\title{
ORDER OF DIRICHLET SERIES WITH REGULAR DISTRIBUTION OF EXPONENTS IN HALF-STRIPS
}

\section{A.M. GAISIN, G.A. GAISINA}

Abstract. We study the Dirichlet series $F(s)=\sum_{n=1}^{\infty} a_{n} e^{\lambda_{n} s}$ with positive and unboundedly increasing exponents $\lambda_{n}$. We assume that the sequence of the exponents $\Lambda=\left\{\lambda_{n}\right\}$ has a finite density; we denote this density by $b$. We suppose that the sequence $\Lambda$ is regularly distributed. This is understood in the following sense: there exists a positive concave function $H$ in the convergence class such that

$$
|\Lambda(t)-b t| \leqslant H(t) \quad(t>0) .
$$

Here $\Lambda(t)$ is the counting function of the sequence $\Lambda$. We show that if, in addition, the growth of the function $H$ is not very high, the orders of the function $F$ in the sense of Ritt in any closed semi-strips, the width of each of which is not less than $2 \pi b$, are equal. Moreover, we do not impose additional restrictions for the nearness and concentration of the points $\lambda_{n}$. The corresponding result for open semi-strips was previously obtained by A.M. Gaisin and N.N. Aitkuzhina.

It is shown that if the width of one of the two semi-strips is less than $2 \pi b$, then the Ritt orders of the Dirichlet series in these semi-strips are not equal.

Keywords: $R$-density of sequence, Dirichlet series, $R$-order, semi-strip, half-plane.

Mathematics Subject Classification: 30D10

\section{INTRODUCTION}

Let $\Lambda=\left\{\lambda_{n}\right\}\left(0<\lambda_{n} \uparrow \infty\right)$ be a sequence obeying the condition

$$
\varlimsup_{n \rightarrow \infty} \frac{\ln n}{\lambda_{n}}=H<\infty .
$$

While studying entire functions defined by everywhere converging Dirichlet series

$$
F(s)=\sum_{n=1}^{\infty} a_{n} e^{\lambda_{n} s} \quad(s=\sigma+i t),
$$

J.F. Ritt introduced the notion of $R$-order. Let us provide the definition of this quantity.

Ritt order ( $R$-order) of an entire function $F$ defined by series $(0.2)$ is the quantity [1]

$$
\rho_{R}=\varlimsup_{\sigma \rightarrow+\infty} \frac{\ln \ln M(\sigma)}{\sigma}, \quad M(\sigma)=\sup _{|t|<\infty}|F(\sigma+i t)| .
$$

We consider a closed strip

$$
S\left(a, t_{0}\right)=\left\{s=\sigma+i t:\left|t-t_{0}\right| \leqslant a\right\}
$$

A.M. Gaisin, G.A. Gaisina, The order of a Dirichlet series with a regular distribution of THE EXPONENTS IN THE HALF-STRIPS.

(c) A.M. Gaisin, G.A. Gaisina 2018.

The work is supported by RFBR (grant no. 17-41-02 0070 r_a).

Submitted July 27, 2018. 
and we denote

$$
M_{s}(\sigma)=\max _{\left|t-t_{0}\right| \leqslant a}|F(\sigma+i t)|
$$

The quantity

$$
\rho_{s}=\varlimsup_{\sigma \rightarrow+\infty} \frac{\ln ^{+} \ln M_{s}(\sigma)}{\sigma} \quad\left(a^{+}=\max (a, 0)\right)
$$

is called $R$-order of a function $F$ in the strip $S\left(a, t_{0}\right)$.

Let

$$
\varlimsup_{n \rightarrow \infty} \frac{n}{\lambda_{n}}=D<\infty, \quad D^{*}=\varlimsup_{\lambda \rightarrow+\infty} \frac{1}{\lambda} \int_{0}^{\lambda} D(x) d x
$$

where $D(x)=\frac{\Lambda(x)}{x}, \Lambda(x)=\sum_{\lambda_{n} \leqslant x} 1 ; D$ is the upper density and $D^{*}$ is the averaged upper density of the sequence $\Lambda$. We obviously have $D^{*} \leqslant D$.

It was proved in [2] that if

$$
\varliminf_{n \rightarrow \infty}\left(\lambda_{n+1}-\lambda_{n}\right)=h>0,
$$

then as $a>\pi D^{*}$, the $R$-order $\rho_{s}$ of the function $F$ in the strip $S\left(a, t_{0}\right)$ is equal to the $R$ order $\rho_{R}$ in the entire plane. The most general result on coinciding $R$-orders in different strip $S_{i}=S\left(a_{i}, t_{i}\right)(i=1,2)$ was established by A.F. Leontiev in [3].

We are going to recall this result. Let $F$ be the sum of Dirichlet series (0.2) and $S_{1}$ and $S_{2}$ be open horizontal strip containing respectively $\bar{D}\left(\alpha_{1}\right)$ and $\bar{D}\left(\alpha_{2}\right)$, where $\bar{D}(\alpha)$ is the shift of the conjugate diagram $\bar{D}$ of the entire function

$$
L(z)=\prod_{n=1}^{\infty}\left(1-\frac{z^{2}}{\lambda_{n}^{2}}\right) \quad(z=x+i y)
$$

by a vector $\alpha$. Then the $R$-orders of the function $F$ in these strip coincide [3, Ch. II, Sect. 5 , Subsect. 3]. For closed strip such result was proved by G.S. Sadykhov in [4].

We note that in [5], for entire Dirichlet series (0.2) of both arbitrary and prescribed order there was made an attempt to obtain the relation

$$
\ln M(\sigma) \sim \ln M_{S}(\sigma)
$$

in the general case as $\sigma \rightarrow \infty$ outside some set $E \subset \mathbb{R}_{+}$, where $\mathbb{R}_{+}$is the positive semi-axis $(0, \infty)$. This relation follows a statement in [5] $]^{1}$; if $\sum_{n=1}^{\infty} \lambda_{n}^{-1}<\infty$ as $F$ has an arbitrary growth or $n=o\left(\lambda_{n}\right)$ as $n \rightarrow \infty$ if $F$ is an entire function of a finite Ritt order, then for each horizontal strip $S_{1} \subset S_{2}$ as $\sigma \rightarrow \infty$ outside some set $E$ of a finite or zero density respectively we have

$$
\ln M_{S_{2}}(\sigma) \geqslant \ln M_{S_{1}}(\sigma) \geqslant \ln \left\{M_{S_{2}}(\sigma)-|o(1)| \mu(\sigma)\right\}+o(\ln M(\sigma)),
$$

where $\mu(\sigma)$ is the maximal term of the Dirichlet series.

However, the expression in curly brackets in (0.4) is generally speaking negative. But then the right estimate in (0.4) makes no sense despite the exponents of the series obey rather strict restrictions, Fejér or Fabri conditions. Nevertheless, if the coefficients $a_{n}$ of series (0.2) lie in a fixed angle $\left\{s=r e^{i \theta}:|\theta| \leqslant \gamma<\frac{\pi}{2}\right\}$, then $|F(\sigma)| \geqslant M(\sigma) \cos \gamma$ and under an appropriate choice of the strip $S_{2}$ relation (0.3) follows easily (0.4). We observe that this condition on $a_{n}$ is not essential, for more details see [7], [8], where stronger results were obtained. The Fejér and

\footnotetext{
${ }^{1}$ A similar result in terms of the coefficients in the Newton majorant was provided in [6] for Dirichlet series converging only in the half-plane.
} 
Fabri conditions are also not essential. ${ }^{1}$ For instance, in [7] the series $\sum_{n=1}^{\infty} \lambda_{n}^{-1}$ was not assumed to be convergent even if $F$ has an arbitrarily high growth rate. Only the finiteness of a so-called $W$-density $G(W)$ was assumed, where $W$ is a convergence class and a condition of type

$$
-\ln h_{n} \leqslant w\left(\lambda_{n}\right) \quad(n \geqslant 1), \quad w \in W,
$$

where $h_{n}=\min _{k \neq n}\left|\lambda_{n}-\lambda_{k}\right|$ was supposed as well. Then, for instance, for each strip $S\left(a, t_{0}\right)$ with $a>\pi G(W)$ relation (0.3) outside a set $E$ of a finite measure [7].

For entire Dirichlet series of finite Ritt order, in [8] there was even proved a criterion of relation (0.3), namely, the sequence $\Lambda$ should have a zero $\alpha$-condensation and satisfy a weaker growth condition than Farbi condition.

In paper [9] the aforementioned by result by A.F. Leontiev in [3] on coinciding Ritt orders in open strips containing $\bar{D}$ was extended to the case, when the convergence domain of series (0.2) is the half-plane $\Pi_{0}=\{s=\sigma+i t: \sigma<0\}$.

Assuming $H=0$ in (0.1), as in [9], we denote by $D_{0}(\Lambda)$ the class of all analytic functions represented by Dirichlet series (0.2) converging only in the half-plane $\Pi_{0}$. In the present work we also consider a subclass of functions in $D_{0}(\Lambda)$ having a finite order similar to the Ritt order in the classical case. The technique and ideas of work [7], where series of arbitrary growth were treated, turn out to be applicable also in the present case [9].

Let $S\left(a, t_{0}\right)=\left\{s=\sigma+i t:\left|t-t_{0}\right| \leqslant a, \sigma<0\right\}$ be a closed half-strip. The quantities

$$
\rho_{R}=\varlimsup_{\sigma \rightarrow 0-} \frac{\ln ^{+} \ln M(\sigma)}{|\sigma|^{-1}}, \quad \rho_{s}=\varlimsup_{\sigma \rightarrow 0-} \frac{\ln ^{+} \ln M_{s}(\sigma)}{|\sigma|^{-1}}
$$

are called Ritt orders of a function $F$ in the half-plane $\Pi_{0}$ and the half-plane $S\left(a, t_{0}\right)[10]$. In what follows, we call the quantities $\rho_{R}$ and $\rho_{s}$ simply by orders in the half-plane and half-strip.

It was shown in [8] that if

$$
\lim _{n \rightarrow \infty} \frac{\ln \lambda_{n}}{\lambda_{n}} \ln n=0,
$$

then the order $\rho_{R}$ of each function $F \in D_{0}(\Lambda)$ is

$$
\rho_{R}=\varlimsup_{n \rightarrow \infty} \frac{\ln \lambda_{n}}{\lambda_{n}} \ln ^{+}\left|a_{n}\right|
$$

the above conditions are also necessary [11].

Let the sequence $\Lambda$ have a finite upper density $D, h(\varphi)$ be a growth indicatrix of the function $L(z)$. Then $\tau=h\left( \pm \frac{\pi}{2}\right) \leqslant \pi D^{*}[2]$. It is obvious that $\tau$ is the type of the function $L$.

If

$$
|L(x)| \leqslant e^{g(x)}(x \geqslant 0), \quad \lim _{x \rightarrow+\infty} \frac{g(x) \ln x}{x}=0,
$$

then the order $\rho_{s}$ in the strip $S\left(a, t_{0}\right)$ as $a>\tau$ and the order $\rho_{R}$ of each function $F \in D_{0}(\Lambda)$ in the half-plane $\Pi_{0}$ satisfy the estimates $[10]$

$$
\rho_{s} \leqslant \rho_{R} \leqslant \rho_{s}+q,
$$

\footnotetext{
${ }^{1}$ Usually one considers two independent problems:

1) the sequence of coefficients $A=\left\{a_{n}\right\}$ is arbitrary and obeys only a natural conditions, but subject to a considered problem, conditions on $\Lambda=\left\{\lambda_{n}\right\}$ are imposed;

2 ) the sequence of exponents $\Lambda$ is arbitrary and obeys only a natural conditions but on $A$ one again imposes conditions motivated by a particular problem.

M.N. Sheremeta considered a combined problem imposing conditions on both $A$ and $\Lambda$ and these conditions are very strict.
} 
where

$$
q=q(L)=\varlimsup_{n \rightarrow \infty} \frac{\ln \lambda_{n}}{\lambda_{n}} \ln \left|\frac{1}{L^{\prime}\left(\lambda_{n}\right)}\right|<\infty .
$$

In [10] there was also considered a case, when the width of the strip $S\left(a, t_{0}\right)$ is exactly $2 \tau$. This implies that if $q<\infty$, then the quantities $\rho_{s}$ and $\rho_{R}$ are finite or infinite simultaneously and $\rho_{s}=\rho_{R}$ as $q=0$. In the general case we have $\rho_{s} \neq \rho_{R}$ [12]. However, it was established in [9] that if the width of each half-strip $S_{i}=S\left(a_{i}, t_{i}\right), i=1,2$, exceeds $2 \pi G(R)$, where $G(R)$ is the $R$-density, then the Ritt orders in these hafl-strips are equal.

As in work [4], a natural question arises: under which conditions each function $F$ in $D_{0}(\Lambda)$ in different half-strips of width not less than $2 \pi G(R)$ have the same order?

The aim of the present work is to provide conditions for the sequences $\Lambda$, under which $\rho_{s_{1}}=\rho_{s_{2}}$, where $\rho_{s_{i}}$ are the orders in arbitrary half-strips $S_{i}, i=1,2$, the width of each is not less than $2 \pi b$. This is done in Theorem 1 . As we shall see in the proof, the quantity $q$ in Theorem 1 can be arbitrary but the theorem does not hold as $a_{1}<\pi b<a_{2}$ even if $q<\infty$; we provide an example of such situation.

\section{PRELIMINARIES}

In what follows we shall need some additional distribution densities of the sequence $\Lambda$. Let $\Lambda=\left\{\lambda_{n}\right\}\left(0<\lambda_{n} \uparrow \infty\right)$ be a sequence possessing a finite upper density, $L$ be a class of positive continuous unboundedly increasing on $[0, \infty)$ functions. By $K$ we denote a subclass of functions $h$ in $L$ such that $h(0)=0, h(t)=o(t)$ as $t \rightarrow \infty, \frac{h(t)}{t} \downarrow$ as $t \uparrow \infty$; the derivative $\frac{h(t)}{t}$ decays monotonically as $t>t_{0}$. In particular, if $h \in K$, then $h(2 t) \leqslant 2 h(t), t \geqslant t_{0}$.

The $K$-density of the sequence $\Lambda$ is the quantity [12]

$$
G(K)=\inf _{h \in K} \varlimsup_{t \rightarrow \infty} \frac{\mu_{\Lambda}(\omega(t))}{h(t)}
$$

where $\omega(t)=[t, t+h(t))$ is a semi-interval, $\mu_{\Lambda}(\omega(t))$ is number of points in $\Lambda$ lying in the semi-interval $\omega(t)$.

Let $\Omega=\{\omega\}$ be a family of semi-intervals of form $\omega=[a, b)$. By $|\omega|$ we denote the length of $\omega$. Each sequence $\Lambda=\left\{\lambda_{n}\right\}, 0<\lambda_{n} \uparrow \infty$, generates an integer-valued counting measure $\mu_{\Lambda}$ :

$$
\mu_{\Lambda}(\omega)=\sum_{\lambda_{n} \in \omega} 1, \quad \omega \in \Omega
$$

Let $\mu_{\Gamma}$ be a counting measure generated by the sequence $\Gamma=\left\{\mu_{n}\right\}, 0<\mu_{n} \uparrow \infty$. Then the inclusion $\Lambda \subset \Gamma$ means that $\mu_{\Lambda}(\omega) \leqslant \mu_{\Gamma}(\omega)$ for each $\omega \in \Omega$. In this case we say that the measure $\mu_{\Gamma}$ majorizes the measure $\mu_{\Lambda}$.

By $D(K)$ we denote the infimum of the numbers $b,(0 \leqslant b<\infty)$, for which there exists the measure $\mu_{\Gamma}$ majorizing $\mu_{\Lambda}$ such that for some function $h \in K$ we have

$$
|M(t)-b t| \leqslant h(t) \quad(t \geqslant 0) .
$$

Here $\Lambda=\left\{\lambda_{n}\right\}, \Gamma=\left\{\mu_{n}\right\}, M(t)=\sum_{\mu_{n} \leqslant t} 1$.

Lemma 1 ([12]). The quantities $D(K)$ and $G(K)$ coincides: $D(K)=G(K)$.

We consider also the following classes of functions:

$$
\begin{aligned}
& L_{0}=\{h \in L: h(x) \ln x=o(x)\} \quad \text { as } \quad x \rightarrow+\infty \\
& R=\left\{h \in K: \quad h(x) \ln \frac{x}{h(x)}=o\left(\frac{x}{\ln x}\right), \quad x \rightarrow+\infty\right\} .
\end{aligned}
$$


The literal reproducing of the proof of Lemma 1 shows that $D(R)=G(R)$; here the quantities $D(R)$ and $G(R)$ are defined as above with the only differences that $h \in R$ in (1.1) and (1.2). It was shown in [12] that if a sequence $\Lambda$ has a finite $G(R)$-density, there exists an even entire function $Q$ with a regular in some sense behavior on the real axis. All zeroes of this functions are real and simple and $\Lambda$ is a subset of its zero zero. Taking into consideration this essentially important fact, it was shown in [9] that $\rho_{S_{1}}=\rho_{S_{2}}$ for all half-strips $S_{i}=S\left(a_{i}, t_{i}\right),(i=1,2)$, having the widths greater than $2 \pi G(R)$. The sequence $\Lambda$ can be extended to the sequence $M$ of all positive zeroes of the function $Q$ with the density $b>G(R)$.

In the present work we assume that the sequence $\Lambda$ has the density $b$ and satisfies a condition of type (1.2). Our aim is to find out, under which additional assumptions for the function $h$, relation (1.2) implies the identity $\rho_{S_{1}}=\rho_{S_{2}}$ for all closed half-strips $S_{1}$ and $S_{2}$ of width at least $2 \pi b$.

We shall need the following theorem.

Lemma 2 ([13]). Let $C(x)$ be a non-decaying function vanishing in the vicinity of zero. Assume that

$$
\int_{0}^{\infty} \frac{C(x)}{x^{2}} d x<\infty
$$

and for $a>0$ we let

$$
m(a)=\int_{0}^{\infty} \frac{C(x)}{x^{2}} d x .
$$

Let $p$ and $q$ be two constants such that $p>2,0 \leqslant q<p-2$. Then for each a there exists an even entire function $F_{a}(z), z=x+i y$, satisfying the conditions:

$$
\left|F_{a}(z)\right| \leqslant e^{p e m(a)|y|-C(|z|)} L_{a}(x, y),
$$

where

$$
L_{a}(x, y)=\frac{\sqrt{\beta \gamma}}{\pi} \frac{1}{1+\beta \gamma\left(x^{2}+y^{2}\right)} .
$$

It is clear that $\left\|L_{a}\right\|_{L(\mathbb{R})}=\left\|L_{a}\right\|_{L(i \mathbb{R})}=1$.

In this lemma

$$
\beta=\frac{(p-q-2) \operatorname{em}(a)}{2}, \quad \gamma=\frac{(p+q) \operatorname{em}(a)}{2},
$$

and the function $F_{a}(z)$ is of the form:

where

$$
F_{a}(z)=\frac{e^{-C(a-0)}}{2 \pi e} \sqrt{\beta \gamma} \frac{\sin \beta z}{\beta z} \frac{\sin \gamma z}{\gamma z} \varphi(z),
$$

$$
\varphi(z)=\prod_{n=1}^{\infty} \frac{\sin \mu_{n} z}{\mu_{n} z} \quad\left(\mu_{n}>0\right)
$$

and

$$
\sum_{n=1}^{\infty} \mu_{n} \leqslant e m(a)
$$

In our case $\Lambda=\left\{\lambda_{n}\right\},\left(0<\lambda_{n} \uparrow \infty\right)$, is the set of all positive zeroes of the function $Q$, that is,

$$
Q(z)=L(z)=\prod_{n=1}^{\infty}\left(1-\frac{z^{2}}{\lambda_{n}^{2}}\right) \quad(z=x+i y) .
$$


Let $\gamma_{Q}$ be a function associated in the Borel sense with an entire function $Q$. Then the following lemma holds.

Lemma 3. The function $Q$ satisfies condition (0.6) if and only if

$$
\varlimsup_{\delta \rightarrow 0+} \delta \ln ^{+} \ln \left|\gamma_{Q}(t)\right| \leqslant 0, \quad \delta=|\operatorname{Re} t| .
$$

The necessary condition was proven in [10], and the sufficient condition was established in $[12]$.

Lemma 4. Let a sequence $\Lambda=\left\{\lambda_{n}\right\},\left(0<\lambda_{n} \uparrow \infty\right)$, satisfy the condition

$$
|\Lambda(t)-b t| \leqslant H(t) \quad(t \geqslant 0),
$$

where the function $H$ belongs to the class $K$ and moreover,

$$
\int_{1}^{\infty} \frac{H(t)}{t^{2}} d t<\infty
$$

Then the entire function $Q$ defined by formula (1.3) satisfy the estimates:

1) there exist $A>0, B>0$ such that

$$
\ln |Q(x)| \leqslant A H(|x|) \ln ^{+} \frac{|x|}{\ln (|x|)}+B
$$

on the real axis;

2) on the imaginary axis we have

$$
\ln |Q(i y)| \leqslant \pi b|y|+2 N_{H}(|y|)+\frac{\pi}{2} H(|y|),
$$

where

$$
N_{H}(r)=\left\{\begin{array}{cl}
\int_{\lambda_{1}}^{r} \frac{H(t)}{t} d t & \text { if } r \geqslant \lambda_{1}, \\
0 & \text { if } 0 \leqslant r<\lambda_{1} .
\end{array}\right.
$$

Estimate (1.6) was proved in [14], while (1.7) follows immediately the representation

$$
\ln |Q(i y)|=2 y^{2} \int_{0}^{\infty} \frac{\Lambda(t) d t}{t\left(t^{2}+r^{2}\right)}
$$

In Lemma 2, we replace $z$ by $i z$ and we let

$$
C(y)=\left\{\begin{array}{cll}
2 N_{H}(y)+\frac{\pi}{2} H(y)+\sqrt{y} & \text { if } \quad y \geqslant \lambda_{1} \\
0 & \text { if } \quad 0 \leqslant y<\lambda_{1} .
\end{array}\right.
$$

Thanks to the term $\sqrt{y}$ we obviously have: $\ln C(y) \asymp \ln y$; we write $\varphi_{1}(y) \asymp \varphi_{2}(y)$ if for some $c_{1}>0, c_{2}>0$ the estimates hold: $c_{1} \varphi_{1}(y) \leqslant \varphi_{2}(y) \leqslant c_{2} \varphi_{1}(y)$. Since the function $N_{H}$ also satisfies condition (1.5), then the function $C(y)$ satisfies the assumptions of Lemma 2. Then combining Lemmata 2 and 4, we obtain the following statement.

Lemma 5. Let $Q$ be the function (1.3), $Q_{a}(z)=F_{a}(i z) Q(z)$, where $F_{a}$ is the function from Lemma 2. Then for each $a>0$, the function $Q_{a}$ satisfies the following estimates on the real and imaginary axes:

1) $\left|Q_{a}(x)\right| \leqslant \exp \left[A H(|x|) \ln ^{+} \frac{|x|}{H(|x|)}+\right.$ pe $\left.m(a)|x|-C(|x|)+B\right] L_{a}(x, 0)$; 
2) $\left|Q_{a}(i y)\right| \leqslant Q\left(i \lambda_{1}\right) L_{a}(0, y) e^{\pi b|y|}$

3) the function $\gamma_{a}$ associated with the function $Q_{a}$ in the Borel sense is continuous on the straight lines $l_{ \pm}=\{t:|\operatorname{Im} t|= \pm \pi b\}$ and

$$
\sup _{|\operatorname{Im} t|= \pm \pi b}\left|\gamma_{a}(t)\right| \leqslant Q\left(i \lambda_{1}\right) \text {. }
$$

All parameters in estimates 1) - 3) are defined in Lemmata 2 and 4.

The estimate in Statement 3) is implied by inequality 2) and the identity $\left\|L_{a}\right\|_{L(i \mathbb{R})}=1$. The continuity of $\gamma_{a}$ on the straight lines $l_{ \pm}$is a well-known fact, see, for instance, [3, Ch. III, Sect. $7])$.

\section{MAIN RESULT}

Let $L_{0}, R$ be the classes of functions introduced above and $G(R)$ be the $R$-density of the sequence $\Lambda$. The following theorem holds true.

Theorem 1. Let $\Lambda=\left\{\lambda_{n}\right\},\left(0<\lambda_{n} \uparrow \infty\right)$, be a sequence obeying condition (1.4) and

$$
\lim _{a \rightarrow \infty} \ln a \int_{a}^{\infty} \frac{N_{H}(x)}{x^{2}} d x=0 .
$$

Given half-strips $S_{1}=S\left(a_{1}, t_{1}\right), S_{2}=S\left(a_{2}, t_{2}\right)$

$$
S\left(a_{i}, t_{i}\right)=\left\{s=\sigma+i t:\left|t-t_{i}\right| \leqslant a_{i}, \quad \sigma<0\right\}, \quad(i=1,2),
$$

of widths at least $2 \pi b$, for each function $F \in D_{0}(\Lambda)$ we have $\rho_{S_{1}}=\rho_{S_{2}}$. Here $\rho_{S_{1}}$ and $\rho_{S_{2}}$ are $R$-orders of the function $F$ in $S_{1}$ and $S_{2}$, respectively.

In the proof of Theorem 1 we shall make use one more statement, namely, the estimate for the quantity $m(\varphi)=-\ln \left|Q\left(r e^{i \varphi}\right)\right|$ as $\varphi \rightarrow 0$, where $Q$ is Weierstrass product (1.3), whose sequence of zeroes $\Lambda=\left\{\lambda_{n}\right\}\left(0<\lambda_{n} \uparrow \infty\right)$ has a positive density $b$ and for some function $H \in R$ we have

$$
|\Lambda(t)-b t| \leqslant H(t), \quad \Lambda(t)=\sum_{\lambda_{n} \leqslant t} 1 .
$$

Lemma 6 ([14]). Under condition (2.1), the entire function $Q$ satisfies the following estimate: there exists $\rho \geqslant 0$ such that as $r \geqslant \rho$, for all $\varphi, 0<|\varphi| \leqslant \frac{\pi}{4}$ we have

$$
|\ln | Q\left(r e^{i \varphi}\right)|-\pi b| \sin \varphi|r| \leqslant 6 H(r) \ln \frac{r}{H(r)}+\frac{8 \pi}{|\varphi|} \frac{H^{2}(r)}{r}+3 \lambda_{1} \tau .
$$

\section{Proof of Theorem 1}

We have

$$
|\Lambda(t)-b t| \leqslant H(t) \quad(t \geqslant 0), \quad H \in R,
$$

and the averaged function $N_{H}$ satisfies condition (1.8). Then an entire function

$$
Q(z)=\prod_{n=1}^{\infty}\left(1-\frac{z^{2}}{\lambda_{n}^{2}}\right) \quad(z=x+i y)
$$

of exponential type $\pi b$ possesses the following properties [14]:

$1^{0} . Q\left(\lambda_{n}\right)=0, \quad Q^{\prime}\left(\lambda_{n}\right) \neq 0 \quad(n \geqslant 1)$;

$2^{0} . \ln |Q(x)| \leqslant g(x) \quad(x \geqslant 0), \quad g \in L_{0}$. 
Moreover, on some sequence of circumferences

$$
K_{n}=\left\{\lambda: \quad|\lambda|=r_{n}\right\}, \quad \frac{r_{n}}{r_{n+1}} \rightarrow 1, \quad n \rightarrow \infty,
$$

the estimates hold true [3, Ch. I, Sect. 3, Subsect. 1]:

$$
\ln |Q(z)| \geqslant-V_{0}(r), \quad r=|z|=r_{n},
$$

where $0<V_{0}(r)=o(r)$ as $r \rightarrow \infty$. Without loss of generality we assume that $n=o\left(r_{n}\right)$ as $n \rightarrow \infty$. In order to see this, for instance, we can exclude some points from the sequence $\left\{r_{n}\right\}$ keeping in each semi-interval $\left[n^{2},(n+1)^{2}\right)$ at most one term of the initial sequence. At that, we choose $r_{1}$ so that $0<r_{1}<\min \left(1, \lambda_{1}\right)$. Taking this into consideration, by $\Gamma_{n}$ we denote a closed contour formed by the arcs of the circumferences $K_{n}=\left\{\lambda:|\lambda|=r_{n}\right\}$, $K_{n+1}=\left\{\lambda: \quad|\lambda|=r_{n+1}\right\}$ and by the segments in the rays $\left\{\lambda: \arg \lambda= \pm \varphi_{n}, 0<\varphi_{n}<\frac{\pi}{4}\right\} ;$ we shall choose $\varphi_{n}$ later.

The following representation holds:

$$
F(s)=\sum_{n=1}^{\infty}\left(\int_{\Gamma_{n}} \frac{\omega_{Q}(\mu, \alpha, F)}{Q(\mu)} e^{\mu s} d \mu\right), \quad s=\sigma+i t \in \Pi_{0},
$$

where $F$ is the sum of the Dirichlet series (0.2) in the class $D_{0}(\Lambda), Q$ is entire function (3.2), and

$$
\omega_{Q}(\mu, \alpha, F)=e^{-\mu \alpha} \frac{1}{2 \pi i} \int_{C} \gamma_{Q}(t)\left(\int_{\alpha_{0}}^{t} F(t+\alpha-\eta) e^{\mu \eta} d \eta\right) d t
$$

is the Leontiev interpolating function, see, for instance, [3, Ch. I, Sect. 2, Subsect. 13]. In the definition of the function $\omega_{Q}(\mu, \alpha, F)$, by $C$ we denote a closed Jordan contour enveloping the conjugate diagram of the entire function $Q, \gamma_{Q}$ is the function associated with $Q$ in the Borel sense, and $\alpha, \alpha_{0}$ are complex parameters. If for instance, the contour $C$ is star-shaped with respect to the origin, one usually lets $\alpha_{0}=0$. In this case $\eta$ in the internal integral in (3.5) ranges in the segment $[0, t]$. Then $t-\eta$ also ranges in the same segment and $(t-\eta) \in \bar{G}$, where $\bar{G}$ is the closure of the domain $G$ bounded by the contour $C$. Then $(t+\alpha-\eta) \in \bar{G}_{\alpha}, \bar{G}_{\alpha}$ is the shift of $\bar{G}$ by a vector $\alpha$. In view of this reason, the parameter $\alpha$ in (3.5) is chosen so that the function $F$ is regular in $\bar{G}_{\alpha}[3]$.

We proceed to proving the identity $\rho_{S_{1}}=\rho_{S_{2}}$. Let $a_{1}, a_{2}$ be arbitrary numbers, $a_{1} \geqslant \pi b$, $a_{2} \geqslant \pi b$, and $S_{1}=S\left(a_{1}, t_{1}\right)$ and $S_{2}=S\left(a_{2}, t_{2}\right)$ be half-strips. We let

$$
a=\sup _{n \geqslant 1} \frac{r_{n+1}}{r_{n}}, \quad c=\left|t_{1}\right|+\left|t_{2}\right|+a_{1}+a_{2}, \quad \varphi_{n}=\varepsilon_{0} \frac{H\left(r_{n}\right)}{r_{n}} \quad(n \geqslant 1) .
$$

Since $H \in R$, then $\varphi_{n} \downarrow 0$ as $n \rightarrow \infty$. We choose number $\varepsilon_{0}$ so that $0<\varphi_{n}<\frac{\pi}{4}(n \geqslant 1)$.

Taking into consideration the identity [15, Ch. IV, Sect. 2, Subsect. 2]

$$
\frac{\omega_{Q}(\mu, \alpha, F)}{Q(\mu)}=\frac{\omega_{Q_{a}}(\mu, \alpha, F)}{Q_{a}(\mu)},
$$

where $Q_{a}$ is the function from Lemma 6 , and introducing a simplified notation $\omega_{a}$ instead of $\omega_{Q_{a}}$, for each $s=\sigma+i t \in \Pi_{0}$ we get:

$$
F(s)=\sum_{n=1}^{\infty}\left(\int_{\Gamma_{n}} \frac{\omega_{a}(\mu, \alpha, F)}{Q_{a}(\mu)} e^{\mu s} d \mu\right)
$$


where

$$
\omega_{a}(\mu, \alpha, F)=e^{-\alpha \mu} \frac{1}{2 \pi i} \int_{C} \gamma_{a}(t)\left(\int_{0}^{t} F(t+\alpha-\eta) e^{\mu \eta} d \eta\right) d t
$$

$\gamma_{a}=\gamma_{a}(t)$ is the function associated with the entire function $Q_{a}$ in the Borel sense, $C$ is a closed convex contour enveloping the conjugate diagram $\bar{D}_{a}$ of the function $Q_{a}, \alpha$ is an arbitrary complex parameter chosen so that $C_{\alpha} \subset \Pi_{0}, C_{\alpha}$ is the shift of $C$ by the vector $\alpha$.

Let us specify the choice of the parameter $\alpha$ and the contour $C$. Let $\gamma_{2} \in(0,1), \gamma_{1}=2 \gamma_{2}^{2}$ and $\alpha=-\sigma\left(1-\gamma_{2}\right)+i t_{1}, \sigma=\operatorname{Re} s<0$. As the estimate in Lemma show, $\bar{D}_{a}$ is contained in the rectangle

$$
\left\{z=x+i y:|x| \leqslant h_{a}(0) \leqslant p e m(a),|y| \leqslant h_{a}\left( \pm \frac{\pi}{2}\right)=\pi b\right\}
$$

where $h_{a}(\varphi)$ is the growth indicatrix of the function $Q_{a}$. As $C$, we take the boundary of the rectangle $P=\left\{z:|\operatorname{Re} z| \leqslant 2\right.$ e em $\left.(a),|\operatorname{Im} z| \leqslant a_{1}\right\}$, where $a_{1}=\pi b$. Taking into consideration that $m(a)$ is a continuous function, $m(a) \downarrow 0$ as $a \rightarrow \infty$, we choose the parameter $a>0$ as the root to the equation

$$
2 p \operatorname{er}(a)=\gamma_{1}|\sigma|
$$

Hence, for $z \in P$ we obtain: $|\operatorname{Re} z| \leqslant \gamma_{1}|\sigma|$. For the convenience we assume that $\gamma_{1}|\sigma| \leqslant 1$.

Our aim is to estimate $|F(s)|$ in the half-strip $S_{2}$ by the maximum of the absolute value of the function $F$ in the half-strip $S_{1}$ by employing some representation of form (3.7). The problem is that $V_{0} \notin L_{0}$ in estimate (3.3). In order to overcome this difficulty, we first prove a lemma.

Lemma 7. Let $s=\sigma+i t \in S_{2}, \mu \in \Gamma_{n}, \eta \in C, \gamma_{2}$ be an arbitrary but fixed number in $(0,1)$. Then

$$
\left|e^{-\mu(\alpha-s-\eta)}\right| \leqslant A\left(\gamma_{2}\right) e^{-\gamma_{2}\left(1+\gamma_{2}\right) r_{n}|\sigma|+a c H\left(r_{n}\right)} \quad(n \geqslant 1),
$$

a, $c$ are above introduced numbers and $H \in R$.

Proof. Letting $\eta=\eta_{1}+i \eta_{2}$, we have

$$
\alpha-s-\eta=-\gamma_{2} \sigma-\eta_{1}-i\left(-t_{1}+t+\eta_{2}\right) .
$$

If $\mu=r e^{i \varphi}=\mu_{1}+i \mu_{2}, R=\operatorname{Re}[-\mu(\alpha-s-\eta)]$, then

$$
R=\mu_{1} \gamma_{2} \sigma+\mu_{1} \eta_{1}-\mu_{2}\left(-t_{1}+t+\eta_{2}\right) .
$$

This implies that

$$
R \leqslant-r \gamma_{2}|\sigma| \cos \varphi+r \gamma_{1}|\sigma| \cos \varphi+r|\sin \varphi| c, \quad r_{n} \leqslant r \leqslant r_{n+1}, \quad 0<|\varphi| \leqslant \varphi_{n}<\frac{\pi}{4} .
$$

Hence,

$$
R \leqslant-r_{n} \gamma_{2}|\sigma|\left(1+2 \gamma_{2}\right) \cos \varphi_{n}+c \varphi_{n} r_{n+1}, \quad n \geqslant 1 .
$$

Since $r_{n+1} \leqslant a r_{n}(n \geqslant 1), \varphi_{n} \downarrow 0$ as $n \rightarrow \infty$, then

$$
R \leqslant-\gamma_{2}\left(1+\gamma_{2}\right) r_{n}|\sigma|+a c H\left(r_{n}\right)
$$

as $n \geqslant n_{0}\left(\gamma_{2}\right)$ and this proves estimate (3.9).

We return back to proving Theorem 1. For $s \in S_{2}, \mu \in \Gamma_{n}$ we are going to estimate the expression

Since $\gamma_{1}|\sigma| \leqslant 1$, then

$$
\left|\frac{\omega_{a}(\mu, \alpha, F) e^{\mu s}}{Q_{a}(\mu)}\right|
$$

$$
\left|\omega_{a}(\mu, \alpha, F) e^{\mu s}\right| \leqslant\left(1+a_{1}^{2}\right)\left|e^{-\mu(\alpha-s)}\right| \max _{\eta \in P}\left|e^{\mu \eta}\right| \max _{t \in C}\left|\gamma_{a}(t)\right| \max _{u \in C_{\alpha}}|F(u)| .
$$


Since the maximum $\max _{\eta \in P}\left|e^{\mu \eta}\right|$ is attained on the contour $C$, by applying Lemma 7 we get:

$$
\left|\omega_{a}(\mu, \alpha, F) e^{\mu s}\right| \leqslant B\left(\gamma_{2}\right) e^{-\gamma_{2}\left(1+\gamma_{2}\right) r_{n}|\sigma|+a c H\left(r_{n}\right)} \max _{t \in C}|\gamma(t)| \max _{u \in C_{\alpha}}|F(u)| .
$$

Here $B\left(\gamma_{2}\right)=\left(1+a_{1}^{2}\right) A\left(\gamma_{2}\right), \mu \in \Gamma_{n},(n \geqslant 1), \gamma_{2} \in(0,1)$.

Taking into consideration relation (3.8), by estimate 1$)$ in Lemma 5 we obtain that

$$
\max _{\substack{t \in C \\|\operatorname{Re} t|=\gamma_{1}|\sigma|}}\left|\gamma_{a}(t)\right| \leqslant e^{B} \exp \left[\max _{x \geqslant 0}\left(A H(x) \ln ^{+} \frac{x}{H(x)}-\frac{\gamma_{1}}{2}|\sigma| x\right)\right] .
$$

Since $H \in R$, this implies easily (cf., for instance, [12]) that

$$
\varlimsup_{|\operatorname{Re} t| \downarrow 0}|\operatorname{Re} t| \ln ^{+} \ln \left|\gamma_{a}(t)\right| \leqslant 0
$$

on the vertical parts of the contour $C$; we observe that for the function $\gamma_{Q}$ associated with $Q$ in the Borel sense, the latter relation is implied by Lemma 3. Therefore, for each , $\gamma_{3}>0$, as $|\sigma|<\varepsilon_{0}=\varepsilon_{0}\left(\gamma_{3}\right)$, on the vertical parts of the contour $C$ we get the estimate

$$
\left|\gamma_{a}(t)\right| \leqslant \exp \exp \left[\gamma_{3} \gamma_{1}^{-1}|\sigma|^{-1}\right], \quad|\operatorname{Re} t|=\gamma_{1}|\sigma|
$$

Since $\left|\gamma_{a}(t)\right| \leqslant Q\left(i \lambda_{1}\right)$ on the horizontal parts of the contour, letting $\gamma_{3}=\gamma_{2}^{4}$ and taking into consideration (3.11) and $\gamma_{1}=2 \gamma_{2}^{2}$, we obtain

$$
\max _{t \in C}\left|\gamma_{a}(t)\right| \leqslant \exp \exp \left[\gamma_{2}^{2}|\sigma|^{-1}\right], \quad|\sigma|<\varepsilon_{1}=\varepsilon_{1}\left(\gamma_{2}\right) \text {. }
$$

Thus, it follows from (3.10), (3.12) for $s \in S_{2}$ and $\mu \in \Gamma_{n}$ that

$$
\left|\omega_{a}(\mu, \alpha, F) e^{\mu s}\right| \leqslant C\left(\gamma_{2}\right) \exp \exp \left[\gamma_{2}^{2}|\sigma|^{-1}\right] e^{-\gamma_{2}\left(1+\gamma_{2}\right) r_{n}|\sigma|+a c H\left(r_{n}\right)} \max _{u \in C_{\alpha}}|F(u)|,
$$

where $\gamma_{1}|\sigma| \leqslant 1,(n \geqslant 1)$.

Since for each $\nu>0$

$$
\left|\frac{\sin \nu z}{\nu z}\right| \geqslant 1
$$

as $|\arg z| \leqslant \frac{\pi}{4}$, then for such $z$

$$
\left|F_{a}(i z)\right| \geqslant \frac{\sqrt{\beta \gamma}}{2 \pi e} e^{-C(a)}, \quad C(a-0)=C(a) .
$$

for each fixed $a>0$.

On arcs of the circumferences $K_{n}$ and $K_{n+1}$ in the contour $\Gamma_{n}$, estimate (3.3) holds. Therefore, in view of the previous estimate, on the same arcs we have

$$
-\ln \left|Q_{a}(z)\right| \leqslant V(r), \quad V(r)=o(r) \quad \text { as } \quad r \rightarrow \infty .
$$

Let $\gamma_{n}$ be the part of the contour $\Gamma_{n}$ without the arcs $C_{n}, C_{n+1},(n \geqslant 2)$, where $C_{n}$ stands for the common part of the contours $\Gamma_{n}$ and $\Gamma_{n+1}(n \geqslant 1)$. We assume that $\gamma_{1}=\Gamma_{1} \backslash C_{1}$, where $C_{1}=\left\{z:|z|=r_{1},|\arg z| \leqslant \varphi_{1}\right\}$. In view (3.13), (3.14) we see that for each fixed $s \in S_{2}$ we have

$$
\left|\frac{\omega_{a}(\mu, \alpha, F) e^{\mu s}}{Q_{a}(\mu)}\right| \leqslant e^{-\gamma_{2}|\sigma| r_{n}}, \quad \mu \in \gamma_{n}, \quad n \geqslant n_{1} .
$$

Hence, for each fixed $s \in S_{2}$,

$$
I_{n}=\int_{C_{n}} \frac{\omega_{a}(\mu, \alpha, F) e^{\mu s}}{Q_{a}(\mu)} d \mu \rightarrow 0
$$


as $n \rightarrow \infty$. And since

$$
\sum_{k=1}^{n}\left(\int_{\Gamma_{k}} \frac{\omega_{a}(\mu, \alpha, F) e^{\mu s}}{Q_{a}(\mu)} d \mu\right)=\sum_{k=1}^{n}\left(\int_{\gamma_{k}} \frac{\omega_{a}(\mu, \alpha, F) e^{\mu s}}{Q_{a}(\mu)} d \mu\right)+I_{n}
$$

instead of (3.6), the following representation is satisfied in half-strip $S_{2}$ :

$$
F(s)=\sum_{n=1}^{\infty}\left(\int_{\gamma_{n}} \frac{\omega_{a}(\mu, \alpha, F) e^{\mu s}}{Q_{a}(\mu)} d \mu\right) .
$$

We first estimate $|Q(\mu)|$ on $\gamma_{n}$ from below uniformly in $\varphi, \varphi_{n+1} \leqslant|\varphi| \leqslant \varphi_{n}$. In order to do this, we employ Lemma 7, according to which, there exists $\rho>0$ such that

$$
-\ln \left|Q\left(r e^{i \varphi}\right)\right| \leqslant 6 H(r) \ln \frac{r}{H(r)}+\frac{8 \pi}{|\varphi|} \frac{H^{2}(r)}{r}+3 \lambda_{1} b
$$

as $r \geqslant \rho$, where

$$
r_{n} \leqslant r \leqslant r_{n+1}, \quad \frac{r_{n+1}}{r_{n}} \rightarrow 1 \quad \text { as } n \rightarrow \infty, \quad r_{n+1} \leqslant a r_{n}, \quad n \geqslant 1, \quad \varphi_{n+1} \leqslant|\varphi| \leqslant \varphi_{n},
$$

$b$ is the density of the sequence $\Lambda=\left\{\lambda_{n}\right\}, a_{1}=\pi b$. Since

$$
H(r) \uparrow \infty, \quad \frac{H(r)}{r} \downarrow 0 \quad \text { as } \quad r \uparrow \infty, \quad \varphi_{n}=\varepsilon_{0} \frac{H\left(r_{n}\right)}{r_{n}},
$$

as $n \geqslant n_{2}$, for $\mu=r e^{i \varphi} \in \gamma_{n}$ we have:

$$
\begin{aligned}
& \text { 1) } 6 H(r) \ln \frac{r}{H(r)} \leqslant 12 H\left(r_{n}\right) \ln \frac{r_{n}}{H\left(r_{n}\right)}, \\
& \text { 2) } \frac{8 \pi}{|\varphi|} \frac{H^{2}(r)}{r} \leqslant \frac{16 \pi}{\varepsilon_{0}} H\left(r_{n}\right) .
\end{aligned}
$$

Thus, in view of the above lower bound for $\left|F_{a}(i z)\right|$ in the angle $\left\{z:|\arg z| \leqslant \frac{\pi}{4}\right\}$, we have:

$$
-\ln \left|Q_{a}(\mu)\right| \leqslant 12 H\left(r_{n}\right) \ln \frac{r_{n}}{H\left(r_{n}\right.}+\frac{16 \pi}{\varepsilon_{0}} H\left(r_{n}\right)+C(a)+\ln \frac{2 \pi e}{\sqrt{\beta \gamma}} \quad\left(n \geqslant n_{2}\right)
$$

on the contour $\gamma_{n}$. Since $H \in R$, the function $H(r) \ln \frac{r}{H(r)}$ belongs to $L_{0}$. Therefore, by (3.13), (3.16) we finally have

$$
\begin{aligned}
\left|\frac{\omega_{a}(\mu, \alpha, F) e^{\mu s}}{Q_{a}(\mu)} e^{\mu s}\right| \leqslant & D\left(\gamma_{2}\right) e^{C(a)} \exp \exp \left[\gamma_{2}^{2}|\sigma|^{-1}\right] \\
& \cdot \exp \left[-\gamma_{2}\left(1+\gamma_{2}\right) r_{n}|\sigma|+w\left(r_{n}\right)\right] \max _{u \in C_{\alpha}}|F(u)|
\end{aligned}
$$

where $w$ is a some function in $L_{0}, \gamma_{1}|\sigma| \leqslant 1, \mu \in \gamma_{n},(n \geqslant 1)$.

Now we can estimate $M_{s_{2}}(\sigma)$ by $M_{s_{1}}(\sigma)$ from above. By (3.15) and (3.17) we obtain:

$$
\begin{aligned}
M_{s_{2}}(\sigma) & =\max _{\left|t-t_{2}\right| \leqslant a_{2}}|F(\sigma+i t)| \\
& \leqslant D\left(\gamma_{2}\right) e^{C(a)} \exp \exp \left[\gamma_{2}^{2}|\sigma|^{-1}\right] \max _{u \in C_{\alpha}}|F(u)| \sum_{n=1}^{\infty}\left|\gamma_{n}\right| \exp \left[w\left(r_{n}\right)-\gamma_{2} r_{n}|\sigma|\right],
\end{aligned}
$$

where $w \in L_{0},\left|\gamma_{n}\right|$ is the length of $\gamma_{n}$.

We consider the Dirichlet series

$$
\Phi(s)=\sum_{n=1}^{\infty} b_{n} e^{\nu_{n} s}
$$


where $\nu_{n}=\gamma_{2} r_{n}(n \geqslant 1), b_{n}=\left|\gamma_{n}\right| \exp \left[w\left(\frac{\nu_{n}}{\gamma_{2}}\right)\right]$, and $n=o\left(\nu_{n}\right)$ as $n \rightarrow \infty$ according the choice of $r_{n}$. It is obvious that series (3.19) converges absolutely in $\Pi_{0}$, and since $w \in L_{0}$, the order of the function $\Phi$ in the half-plane $\Pi_{0}$ is zero (see [10] and (0.5)):

$$
\rho(\Phi)=\varlimsup_{n \rightarrow \infty} \frac{\ln \nu_{n}}{\nu_{n}} \ln ^{+}\left|b_{n}\right|=0 .
$$

Since according condition (1.8) we have $m(a) \ln a \rightarrow 0$ as $a \rightarrow \infty$, and $\ln C(a) \asymp \ln a$, then in view of identity (3.8) we conclude that $|\sigma| \ln C(a(|\sigma|)) \rightarrow 0$ as $\sigma \uparrow 0$; it is also obvious that $a(|\sigma|) \uparrow \infty$. In view of the above fact, by (3.18) we obtain

$$
M_{s_{2}}(\sigma) \leqslant \max _{u \in C_{\alpha}}|F(u)| \exp \exp \left[2 \gamma_{2}^{2}|\sigma|^{-1}\right], \quad 0<|\sigma|<\varepsilon_{2}\left(\gamma_{2}\right) .
$$

We choose $\gamma_{2} \in\left(0, \frac{1}{2}\right)$. Since $\alpha=|\sigma|\left(1-\gamma_{2}\right)+i t_{1}$, then

$\left|\operatorname{Im} u-t_{1}\right| \leqslant a_{1}, \quad|\sigma|\left(1-\gamma_{2}-\gamma_{1}\right) \leqslant \operatorname{Re} u \leqslant|\sigma|\left(1-\gamma_{2}+\gamma_{1}\right), \quad \gamma_{1}=2 \gamma_{2}^{2}<\gamma_{2} \quad$ as $\quad 0<\gamma_{2}<\frac{1}{2}$

if $u \in C_{\alpha}$. Therefore, if in the half-strip $S_{1}$ the function $F$ has an order equal to $\rho_{s_{1}}$, then by (3.20) we finally have

$$
M_{s_{2}}(\sigma) \leqslant \exp \exp \left[2 \gamma_{2}^{2}|\sigma|^{-1}\right] \exp \exp \left[\left(\rho_{s_{1}}+\gamma_{2}\right)\left(1-\gamma_{2}-\gamma_{1}\right)^{-1}|\sigma|^{-1}\right], \quad 0<|\sigma|<\varepsilon_{3}\left(\gamma_{2}\right) .
$$

This yields

$$
M_{s_{2}}(\sigma) \leqslant \exp \exp \left[\left(\rho_{s_{1}}+3 \gamma_{2}\right)\left(1-\gamma_{2}-\gamma_{1}\right)^{-1}|\sigma|^{-1}\right], \quad 0<|\sigma|<\varepsilon_{4}\left(\gamma_{2}\right) .
$$

This means that the order $\rho_{s_{2}}$ in the half-strip $S_{2}$ satisfies the estimate

$$
\rho_{s_{2}} \leqslant \frac{\rho_{s_{1}}+3 \gamma_{2}}{1-\gamma_{2}-\gamma_{1}}, \quad \gamma_{1}=2 \gamma_{2}^{2}, \quad 0<\gamma_{2}<\frac{1}{2} .
$$

Since $\gamma_{2} \in\left(0, \frac{1}{2}\right)$ is arbitrary, then $\rho_{s_{2}} \leqslant \rho_{s_{1}}$ if $a_{2} \geqslant \pi b, a_{1}=\pi b$, and of course, as $a_{1} \geqslant \pi b$. In the same way we prove the inverse inequality. Thus, $\rho_{s_{1}}=\rho_{s_{2}}$ for all half-strips $S\left(a_{1}, t_{1}\right)$ and $S\left(a_{2}, t_{2}\right)$ if $a_{1} \geqslant \pi b, a_{2} \geqslant \pi b$.

Remark 1. In the proven theorem $G(R)<\infty$ although this statement makes sense also in the case $G(R)=\infty$; we just need to consider the half-strips of form $S\left(\infty, t_{0}\right)$ coinciding with the half-strip $\Pi_{0}$ and then again $\rho_{s_{1}}=\rho_{s_{2}}$. But Theorem 1 is not reduced to the simple case $\rho_{s}=\rho_{R}$, where $\rho_{R}$ is the order of the function $F$ in the half-plane $\Pi_{0}$ calculated by formulae (0.5) via the coefficients, and $\rho_{s}$ is the order in the half-strip $S\left(a, t_{0}\right), a \geqslant \pi b$, see [9], [10].

Remark 2. Under assumptions of Theorem 1 as $b=0$, the identity $\rho_{s_{1}}=\rho_{s_{2}}$ holds for all half-strips $S\left(a_{1}, t_{1}\right), S\left(a_{2}, t_{2}\right), a_{1}>0, a_{2}>0$ are arbitrary. However we note that an analogue of Theorem 1 for horizontal rays is not true [16].

It turns out that as only one of the half-strips has a width less than $2 \pi b$, the theorem is no longer true as well even if we assume that $q<\infty$.

Let us adduce an appropriate example. Let $\Lambda=\left\{\lambda_{n}\right\},\left(0<\lambda_{n} \uparrow \infty\right)$, be an arbitrary sequence with a finite density $b$ satisfying assumptions of Theorem 1.

We let

$$
\psi(\lambda)=\prod_{k=1}^{\infty}\left(1+\frac{\lambda^{2}}{b_{k}^{2}}\right),
$$

where the numbers $b_{k}$ with $k>N$ are determined by the identities

$$
\frac{k}{b_{k}^{\rho\left(b_{k}\right)}}=\Delta, \quad \Delta>0,
$$


and at that, the numbers $b_{k},(k \leqslant N)$, are arbitrary, $0<b_{1}<b_{2}<\ldots<b_{N}, \rho(r)=1-\frac{\ln \ln r}{\ln r}$, $r \geqslant e^{e}$; this is obviously a specified order. An entire function $\psi$ has a minimal type at order 1 and

$$
(\pi \Delta-\varepsilon)|x|^{\rho(|x|)} \leqslant \ln |\psi(x)| \leqslant(\pi \Delta+\varepsilon)|x|^{\rho(|x|)}
$$

on the real axis [15] as $|x| \geqslant r_{0}(\varepsilon), \varepsilon>0$ is arbitrary.

We consider the Dirichlet series

$$
\Psi(s)=\sum_{k=1}^{\infty} \frac{e^{\lambda_{k} s}}{Q^{\prime}\left(\lambda_{k}\right)} \quad(s=\sigma+i t) .
$$

For this series the convergence domain is the half-plane $\Pi_{0}$; since $q<\infty$, the condensation index of the sequence $\Lambda$ is equal to 0 . It is easy to confirm that the integral

$$
I(s)=\frac{1}{2 \pi i} \int_{-\infty i}^{\infty i} \frac{e^{\xi s}}{Q(\xi)} d \xi
$$

converges uniformly inside the strip

$$
S=\{z:|\operatorname{Im} z|<\pi b\}
$$

and defines an analytic in $S$ function $I(s)$ bounded in each strip $\{s=\sigma+i t:|t| \leqslant a<\pi b\}$, $a>0$.

In an usual way one can show that by means of the integral (3.22), the sum of series (3.21) is analytically continued in the strip $S$ through the interval $(-\pi b i, \pi b i)$, see, for instance, [3] and [17, Thm. 2.1.4]. But then the Dirichlet series

$$
F(s)=\sum_{k=1}^{\infty} \frac{\psi\left(\lambda_{k}\right)}{Q^{\prime}\left(\lambda_{k}\right)} e^{\lambda_{k} s}
$$

also converges in the half-plane $\Pi_{0}$ and is continued analytically over all curves along with the function $\Phi$ can be continued [15, Thm. 2.4.1]. Therefore, the function $F$ is bounded in the half-strip $S_{1}=S\left(a_{1}, 0\right),\left(0<a_{1}<\pi b\right)$, and this is why $\rho_{s_{1}}=0$. Its Ritt order $\rho_{R}$ is obviously equal to $\pi \Delta+q$. Since under the assumptions of Theorem 1 , the function $Q$ satisfies conditions (0.6), as it follows from (0.7), for each half-strip $S_{2}=S\left(a_{2}, t_{0}\right)\left(a_{2}>\pi b\right)$ we have $\rho_{s_{2}} \geqslant \rho_{R}-q=\pi \Delta>0$.

On the other hand, as it has been shown in Theorem $1, \rho_{S_{2}}=\rho_{b}$, where $\rho_{b}$ is the order in the half-strip $S\left(b, t_{0}\right)$ and hence, $\rho_{b}>0$.

Theorem 2. Let a sequence $\Lambda$ satisfies assumptions of Theorem 1. Then for each function $F \in D_{0}(\Lambda)$, its orders $\rho_{S_{1}}$ and $\rho_{S_{2}}$ in the half-strips $S\left(a_{1}, t_{1}\right)$ and $S\left(a_{2}, t_{2}\right)$ are equal if and only if the width of each strip is not less than $2 \pi b$.

\section{BIBLIOGRAPHY}

1. J.F. Ritt. On certain points in the theory of Dirichlet series // Amer. J. Math. 50:1, 73-86 (1928).

2. S. Mandelbrojt. Séries adhérentes. Régularisation des suites. Applications. Gauthier-Villars, Paris (1952).

3. A.F. Leont'ev. Sequences of exponential polynomials. Nauka, Moscow (1980). (in Russian).

4. G.S. Sadykhov. Issues on grwoth of functions defined by Dirichlet series and other more general series. Review of PhD thesis, Moscow (1968). (in Russian).

5. M.N. Sheremeta. Growth in a strip of entire functions represented by Dirichlet series // Izv. AN SSSR. Ser. Matem. 45:3, 674-687 (1981). [Math. USSR-Izv. 18:3, 587-598 (1982).] 
6. O.B. Skaskiv. Asymptotic properties of analytic functions represented by power series and Dirichlet series. Review of Habilitation thesis, Lviv (1996). (in Ukrainian).

7. A.M. Gaisin. An estimate for a Dirichlet series whose exponents are zeros of an entire function with irregular behavior // Matem. Sborn. 185:2, 33-56 (1994). [Russ. Acad. Sci. Sb. Math. 81:1, 163-183 (1995)].

8. A.M. Gaisin. Asymptotic properties of functions defined by exponential series. Habilitation thesis, Ufa (1994). (in Russian).

9. A.M. Gaisin, N.N. Aitkuzhina. The order of a Dirichlet series with an irregular distribution of the exponents in the half-strip // // Alg. Anal. 30:4, 27-46 (2018). [St. Petersburg Math. J. (2019), to appear.]

10. A.M. Găsin. A bound for the growth in a half-strip of a function represented by a Dirichlet series // Matem. Sbornik. 117(159):3, 412-424 (1982). [Math. USSR-Sbornik. 43:3, 411-422 (1983).]

11. N.N. Aitkuzhina, A.M. Gaisin. Exactness of estimates for $k$ th order of Dirichlet series in a semistrip // Ufimskij Matem. Zhurn. 7:4, 15-24 (2015). [Ufa Math. J. 7:4, 15-23 (2015).]

12. A.M. Gaisin, D.I. Sergeeva. An estimate for the Dirichlet series in a half-strip in the case of the irregular distribution of exponents. II // Sibir. Matem. Zhurn. 49:2, 280-298 (2008). [Siber. Math. J. 49:2, 222-238 (2008).]

13. S. Mandelbrojt. Dirichlet series. Principles and methods. D. Reidel Publishing Company, Dordrecht (1972).

14. A.M. Gaisin, D.I. Sergeeva. Entire functions with a given sequence of zeros and of regular behavior on the real axis. I // Sibir. Matem. Zhurn. 48:5, 995-1007 (2007). [Siber. Math. J. 48:5, 798-808 (2007).]

15. A.F. Leontiev. Exponential series. Nauka, Moscow (1976). (in Russian).

16. A.M. Gaisin. Behavior of the sum of a Dirichlet series in halfstrips // Matem. Zamet. 42:5, 660-669 (1987). [Math. Notes. 42:5, 863-868 (1987).]

17. I.I. Ibragimov. Interpolation methods for functions and some of their applications. Nauka, Moscow (1971). (in Russian).

Akhtyar Magazovich Gaisin, Institute of Mathematics, Ufa Federal Research Center, RAS,

Chernyshevsky str. 112,

450008, Ufa, Russia,

Bashkir State University,

Zaki Validi str. 32,

450074, Ufa, Russia

E-mail: gaisinam@mail.ru

Galiya Akhtyarovna Gaisina,

Bashkir State University,

Zaki Validi str. 32,

450074, Ufa, Russia

E-mail: gaisinaga@mail.ru 